\section{OPEN ACCESS}

Edited by: Aaron Williamon,

Royal College of Music and Imperial

College London, UK

Reviewed by:

Ronald Hübner,

University of Konstanz, Germany

Garga Chatterjee,

Indian Statistical Institute, India

${ }^{*}$ Correspondence:

Susan Beudt

s.beudt@hsu-hh.de

Thomas Jacobsen

jacobsen@hsu-hh.de

Specialty section:

This article was submitted to

Cognitive Science,

a section of the journal

Frontiers in Psychology

Received: 12 August 2016 Accepted: 19 January 2017

Published: 07 February 2017

Citation: Jacobsen T and Beudt S (2017) Stability and Variability in Aesthetic Experience: A Review. Front. Psychol. 8:143. doi: 10.3389/fpsyg.2017.00143

\title{
Stability and Variability in Aesthetic Experience: A Review
}

\section{Thomas Jacobsen* and Susan Beudt*}

Experimental Psychology Unit, Humanities and Social Sciences, Helmut-Schmidt-University/University of the Federal Armed Forces Hamburg, Hamburg, Germany

Based on psychophysics' pragmatic dualism, we trace the cognitive neuroscience of stability and variability in aesthetic experience. With regard to different domains of aesthetic processing, we touch upon the relevance of cognitive schemata for aesthetic preference. Attitudes and preferences are explored in detail. Evolutionary constraints on attitude formation or schema generation are elucidated, just as the often seemingly arbitrary influences of social, societal, and cultural nature are. A particular focus is put on the concept of critical periods during an individual's ontogenesis. The latter contrasting with changes of high frequency, such as fashion influences. Taken together, these analyses document the state of the art in the field and, potentially, highlight avenues for future research.

Keywords: aesthetics, aesthetic experience, experimental aesthetics, art, cognitive schemata, scripts, attitudes, sensitive period

\section{INTRODUCTION}

In aesthetics, we deal with a multitude of factors influencing our preferences, judgments, contemplation, appreciation, liking, and disliking. While we are searching for laws of aesthetics universal for mankind, there is also no accounting for taste. While Homo sapiens appears to be the only primate species capable of fully developed aesthetic processing, there is also no denying that evolution has an influence on our aesthetic appreciation. While history has demonstrated a wide variety of often seemingly arbitrary ideals of beauty, we, as psychologists, would still hope to be able to identify general underlying mental mechanisms. For the endeavor of tracing stability and variability in aesthetic processing in this article, we will adopt a framework for the psychology of aesthetics that uses several vantage points (Jacobsen, 2006, 2010a). For one, it is based on psychophysics pragmatic dualism, using the mind and brain perspectives. It also holds the view that all episodes of mental processing of art, that is all episodes of aesthetic appreciation for our concern, manifested as an interaction of person and situation variables modified by different content domains. Furthermore, it asserts that changes happen over time (diachronia) and that they take place for different reasons and on differing timescales. Some may happen fast due to rapid changing in social influences, as seen in fashion for instance, while others happen on extremely slow time scales, like changes due to evolutionary biology. While biological universals may have been with the modern man for more than 100,000 years, and would thus be considered very stable, the same anatomy provides for extremely individualized and highly diverse preferences in various domains of aesthetic appreciation.

Aesthetic appreciation entails the evaluation of sensations and perceptions against relevant concepts like the beautiful, the elegant, the harmonious, the melodious, the rhythmical, and the like. These concepts show relative stability over time, over individuals, as well as aesthetic domains. The concept of beauty is central to many domains of aesthetic appreciation. On the other hand, the conceptual structure shows relative variability as well. Rhythm and melody 
are important for music and poems, but less so for faces (Jacobsen et al., 2004; Istók et al., 2009; Augustin et al., 2012; Knoop et al., 2016). For the present review, we have selected two theoretical psychological notions as a basic structure of our analysis, the notion of attitude and the notion of schema or script. Both are long term memory representations that have been shown to influence, if not guide, aesthetic appreciation.

There were various attempts to attain a reasonable definition of the concept of attitudes (for an overview, see e.g., Riemer et al., 2014), but all share the notion of evaluation. For a variety of objects humans have acquired relatively stable "[...] summary evaluations [...] ranging along a dimension [...] from positive to negative" (Petty et al., 1997), so that in sum an attitude object can be linked with liking/disliking. Thus an individual who is about to aesthetically appreciate an (aesthetic) object, will approach it with its own attitudinal system. That system may, for instance, comprise evaluations of one's own expertise with regard to the aesthetic domain or, more specifically, the genre at hand. If information about the creator of the aesthetic object is available, the aforementioned may be implicated with stored evaluations of various groups. In addition, the aesthetic object might address explicitly specific themes or induce associations with specific related issues (even if not intended by the creator) for which summarized evaluations are stored and automatically activated upon exposure with the object.

The schema concept has also been used and described in various ways, mostly based on conceptions suggested by Piaget (1926) and Bartlett (1932). According to Mandler (1984), "knowledge about an object or classes of objects, about an event or classes of events, about personality traits and social norms, can all be considered as small networks of information that become activated as we experience these things". Thus, schemata are higher-order cognitive structures that are relevant to understand how old knowledge and past experiences interact with new information (Brewer and Nakamura, 1984). Referring again to a situation in which an object is aesthetically appreciated, the perceiver not only approaches the aesthetic object with a set of relevant attitudes, but also holds ready applicable schemata. Whereas attitudes often elicit reactions or responses toward the attitude objects, schemata provide frameworks which allow for making sense of the given situation or aesthetic object by adding missing details. Based upon the attributes and their interrelations which are stored for a particular schema, expectations are formed and missing information can be added. Depending on various aspects of the situation, for instance, the type of aesthetic object (e.g., static painting versus dynamic piece of performance art) or the context of the experience (e.g., private house versus museum versus laboratory), different (types of) schematic representations are activated. For aesthetic appreciation self-schemas and event schemas, which are commonly referred to as cognitive scripts, may be foremost important. A script holds information about "appropriate sequences of events in a particular context," is composed of "slots and requirements about what can fill those slots," and due to interconnections "what is in one slot, affects what can be in another" (Schank and Abelson, 1977). For instance, different scripts will be addressed during a museum visit and during a theater play. In sum, the activated set of attitudes, schemata and scripts upon exposure to an aesthetic object may then modulate aesthetic appreciation.

We trace the importance of these mental representations through different domains of aesthetic processing, while we investigate the variability or stability of either the mental representation itself or variability or stability of its contents. Attitudes and schemata/scripts are very frequently acquired implicitly. They are influenced unintentionally, or overlearned intentionally. Their flexibility or plasticity may change over time, may change over the course of ontogeny. Critical periods for either the acquisition or the change of an attitude or a schema may apply. It is possible that while the content of the script is bound to change on a regular, fixed basis and time scale, the script itself has been acquired for life and is thus stable.

There may be domains of aesthetic processing in which individual differences in aesthetic appreciation have to be accounted for in order to be able to identify underlying general mental mechanisms. For instance, the identification of the brain correlates of aesthetic judgment of beauty of novel, formal graphic patterns required the formal modeling of the participants' individual judgments (Jacobsen et al., 2006). That is, while individual beauty ratings for the graphic material presented to the participants were highly heterogeneous, a common neural network subserving the process of aesthetic judgment could be identified.

This review is organized as follows. The first section addresses the role of schemata and scripts in the context of aesthetics. In the second section the role of attitudes and preferences in aesthetic appreciation is explored. This is done by first exploring developmental aspects (e.g., sensitive periods). Subsequently variability and stability of relevant attitudes and aesthetic preferences across different aesthetic domains are elaborated on the basis of respective research accounts.

\section{SCHEMATA/SCRIPTS}

It is, most likely, safe to assume that we all command long-term memory representations of the schema and script type of various aesthetic domains, arts, and art genres. If we have not intended to acquire such a memory representation actively, we have implicitly acquired it.

Implicitly acquired, we most likely command a host of, in part, very diversified schemata and scripts. Besides prescribed actions or rather non-actions, like not touching exhibits in a gallery or a museum, schemata and scripts also entail and prescribe affective responses to art. Taking the conceptual structure of the aesthetics of literature as a starting point, the study by Knoop et al. (2016) shows that comedies as a literary genre prescribes more funny cognition than tragic, for instance, while plays, in general, including drama, are prescribed as tragic, dramatic, and also sad. The data show that, most likely, theater goers as well as readers of drama or comedy command a script of the reception of such a play that also includes elements of an affect script. Going to the cinema planning to watch a sad movie, entails elements of emotion regulation. We know that there will be sad content. Studies by 
Hanich et al. (2014) and Wassiliwizky et al. (2015) demonstrated that a very important emotional response to sad movies is the emotion of being moved. Being emotionally moved by the movie one watches most likely is an element of emotion regulation mediated by the art reception script including elements of an effect script. Scripts of this rather general type appear to be rather stable, although there is no empirical study on a critical period of acquisition and or the stability of these scripts overtime.

Just as other social conventions are acquired over a number of experiences, art schemata of different domains are frequently acquired implicitly. Attending a concert of classical music entails being quietly seated, avoiding making noises, not getting up to dance, not talking to fellow listeners, spending applause only in the right moments in time, and so on and so forth. Attending the showing of a theater play conventionally entails being quietly seated avoiding making noises, not talking to one's neighbor, not getting up to dance, and spending applause only in the right moments. This does, of course, not mean that such a script cannot be broken. As it often occurs in modern theater, audience may be urged to participate, like actually getting up to dance, for instance. In such a moment, viewers may feel insecure and uncomfortable, if not used to participate in things like that. This little anecdote demonstrates that implicitly acquired scripts and schemata may very well have the power to shape our experience, to be real. It also shows that scripts and schemata may contain certain default slots that guide our experience when filled. For instance, in the above mentioned examples of classic concerts and theater plays, the audience is at a safe distance to the performance. In the conventional version of the script, audience participation clearly is not called for. Thus, the members of the audience do not have to be afraid of being exposed to other members. Rather, one can safely assume to be left in the exclusively receptive role. This fact may foster focuses on individual subjective feeling, self-referential appreciation and evaluation of sensation and perception.

Some schemata may actually be quite diversified and detailed, even on the bases of implicit acquisition. Art schemata and scripts are constituted in a social fashion. This means they are subject to change on the bases of social and cultural change. Therefore, stability and variability of art schemata and scripts hinge on cultural and social factors. On the other hand, some very basic schemata may even have evolutionary underpinnings. An example may be the basic narrative scheme, making minimum requirements for a narrative plot. The art form for poetry, for instance, may have characteristics that speak to a coevolution of our language and memory faculties. The more evolutionary grounding an arts schema or art script has, the less likely it should be subject to change, and thus be very stable.

The notion of scripts for the reception of performing arts as introduced by the affect script example above, most likely, extends to various narrative and/or performing art forms. To date, we do not know of systematic studies on the acquisition and the ex-act design of these scripts.

\section{ATTITUDES AND PREFERENCES}

Long-term memory representations such as attitudes are perceived as sets of evaluations stored for an object and ranging over a positive - negative dimension (Petty et al., 1994). A common notion is that attitudes entail a knowledge component, a valence aspect and a behavioral tendency. Differences of their underlying structure are thought to influence their strength, i.e., their persistence over time, resistance against counterpersuasion and thus the degree of their influence on judgment and behavior. Getting activated automatically, those evaluations facilitate a quick assessment of the everyday object being processed (for a review, see Petty et al., 1997).

Representing an important modulator of aesthetic experiences, attitudes may often override other effects for which influences on aesthetic appreciation have been demonstrated (i.e., fluency, familiarity, mere exposure). Theoretical accounts for this modulation are still scarce. Some attention has been drawn to this issue within the framework of automatic and controlled processes of aesthetic appreciation provided by Jacobsen (2010b), in which memory systems operating at different levels of aesthetic appreciation are reviewed.

When stimuli are being accommodated in the perceiver's attitude system, the latter may have a profound influence on the aesthetic appreciation of these stimuli (see Ritterfeld, 2002, for a seminal study or Istók et al., 2013). In fact, attitudinal influences could even lead to outright memory-based judgments of beauty or other important concepts (Jacobsen et al., 2004; Istók et al., 2009; Augustin et al., 2012; Knoop et al., 2016), instead of proper aesthetic processing. Hence, some past research endeavors in the field considered attitudinal influences as confounding factors that needed to be reliably excluded, for instance by using novel stimuli (visual stimuli: e.g., Jacobsen and Höfel, 2002, 2003; Höfel and Jacobsen, 2003, 2007a,b; Jacobsen, 2004a; music stimuli: e.g., Brattico et al., 2010; Kornysheva et al., 2010; Istók et al., 2013) that could less likely be integrated automatically in the perceiver's attitude system on grounds of knowledge and expertise.

One common way to gauge the automatic activation of attitudes is the application of the implicit association test (IAT), which allows an exploration of the unconscious use of the attitudinal memory system (e.g., Fazio and Olson, 2003). By now this approach has been adopted in a few studies within aesthetic domains such as the human body (Ahern et al., 2008), visual art and architecture (Mastandrea et al., 2011), and design objects (Mastandrea and Maricchiolo, 2014). We will now explore findings regarding sensitive periods for the development of attitudes relevant to aesthetic contemplation and we will try to relate those findings to the development of aesthetic preferences for different domains of aesthetic appreciation.

\section{Acquisition and Sensitive Period of Attitudes and Aesthetic Preferences}

Appreciation of art and aesthetics are often considered as being unique for the human species (e.g., Dobzhansky, 1962). During ontogenesis of both the human perceptual system and the human conceptual system, foundations are laid for the capacity of 
aesthetic evaluation and thereby as well for the formation of taste. Several aspects support this notion.

Regarding the development of visual perception, among those aspects is, for instance, the existence of ocular dominance columns (Hubel and Wiesel, 1962), for which a critical period (postnatal up to 5 and more years of age) for their experiencedependent neuroplasticity has been widely investigated (for a review, see e.g., Hooks and Chen, 2007). Another aspect refers to functional specialization of individual neurons as well as of neural circuits for the processing of, for instance, single features (like color or motion direction; see e.g., Zeki et al., 1991) or complex shapes and objects (like faces, substantially processed by the fusiform face area; Kanwisher et al., 1997; Kanwisher and Yovel, 2006). The development of face processing sensitivity has been investigated in a study with subjects, who have been blind from birth but who had their vision restored via cataract surgery (Röder et al., 2013). Due to the varied time periods of blindness among subjects, identification of a closer defined sensitive period has not been possible, though the results could demonstrate that such a period exists. As to sensitive periods for the development of other characteristics of the visual system, different time periods seem to exist, in that, for instance, the development of normal visual acuity and peripheral light sensitivity are impaired when visual deprivation begins at 6 months after birth (review by Lewis and Maurer, 2005). Interestingly, this does not apply for global motion sensitivity for which impairment could only be shown when visual deprivation began near birth (Lewis and Maurer, 2005).

Within the limits of this manuscript, we do not aspire to give an ample overview of the findings regarding sensitizing periods for all perceptual qualities. Preliminary to reviewing findings regarding the development of aesthetic preferences and attitudes toward musical stimuli, we will shortly address sensitive periods for the auditory domain. Similar to the development of the visual perceptual system, for auditory perception the early years after birth are crucial for developing normal auditory feature processing (for reviews, see e.g., Trainor, 2005; Howard-Jones et al., 2012). For instance, the neonate brain's sensitivity to discriminate acoustic differences is initially very high, before it learns to organize auditory input according to distinctive features and to belonging to certain categories while learning to ignore more and more insignificant variability of sounds (for a review, see Kral, 2013). Besides being obviously crucial for language learning (for a review, e.g., Kuhl, 2010), this initial sensitive period is also relevant to musical learning (Penhune, 2011). Young infants are able to discriminate acoustic properties (e.g., timing and pitch, Trehub, 2003) and they are sensitive to processing rhythm, melody, aspects of harmony (Trehub, 2006; Trehub et al., 1997), which is relevant for music processing and therefore for later musical preferences (for a review, see Nieminen et al., 2011).

Whether musical preferences are biologically determined or acquired through exposure is still being debated. Studies investigating musical preferences in neonates and young infants indicate that sensory processing of basic musical properties, along with core liking of those properties (like preference for consonance: Perani et al., 2010), occur early in child development (Nieminen et al., 2011, 2012).

The development of a distinctive musical taste which characterizes an individual has been outlined by Brattico et al. (2013). In their neuro-cognitive model of the aesthetic processing of music they proposed that musical taste results from a long-term set of preferences, attitudes, values and aesthetic judgments and those in turn should be influenced by individual characteristics such as age, gender, social status, and personality. Whether listeners arrive at consciously liking or disliking a musical piece should be determined by an interaction of their musical taste and their context variables (mood, attention, social attitudes). The authors described this as building a feedback loop which should be more pliable within early adulthood if compared to infancy or adulthood (Brattico et al., 2013). In young listeners (daycare and elementary school) music preferences have been shown to be strongly affected by social factors such as preferences of parents and siblings (Roulston, 2006) and for 7- to 8-yearold children exposure to gender stereotypic role models seems to be relevant for choosing a musical instrument (Harrison and O'Neill, 2000). Late adolescence and early adulthood have been linked to individual's increased musical genre exploration, which is in line with the open-earedness hypothesis (LeBlanc et al., 1996; Hargreaves and North, 2010). According to the openearedness hypothesis younger children show more tolerance toward musical styles, which are categorized as unconventional by adults, possibly as a result of being less "adapted to" normative standards with regard to what is considered as good and bad taste in the given culture. Exploring various musical genres in this developmental stage is thought to help shape and crystallize musical taste.

Lately it is discussed, that adolescence may provide also periods for sensitization, mainly concerning higher-order processes (Howard-Jones et al., 2012; Fuhrmann et al., 2015). It was also suggested, that for bi-directional transfer of skills between language and musical processing there may exist a sensitive period as well (White et al., 2013).

\section{Variability and Stability of Attitudes and Aesthetic Preferences}

The notion of a universal in aesthetic appreciation constitutes the strongest variant of stability. Historically and culturally invariant, its traces can be observed in individual behavior. In a psychophysical sense, adopting the mind-brain perspective, taste can be considered a long-term representation that is more or less changing, or the respective bodily substrate. Furthermore, taste also comprises a more generalized element when regarding the scope of a genre or style (for musical domain, see e.g., Istók et al., 2013).

Taste in general is subject to change which happens at different time scales. In fashion, taste once was expected to adapt on a 6 months' cycle. By now this has been taken to the extreme, anecdotally in the context of fast fashion cheaply imitating fashion trends and involving (in the case of some fashion brands) up to 6 mid-seasonal trends per year. There is most likely, although evidence is still scarce, highly stable taste based on 
ontogenetic acquisition during critical periods (as depicted in Chapter 2.1). On the other extreme, there are species universals that are expected to be stable, or at best culturally modified. Research investigating universal, biologically based aesthetic tendencies and their shaping by cultural and historical influences (see ipsichronia vantage point, Jacobsen, 2006) is scarce so far (for an overview on cross-cultural differences and similarities regarding attractiveness of faces or body features, see Little et al., 2011).

One factor that is regarded as a standard in aesthetic appreciation shared across cultures is symmetry, which seems to be one of the biologically based aspects of beauty. Though within culture perspective there exist accounts of more variability across aesthetic domains. For the aesthetics of faces similar aesthetic tendencies across different cultures have been found (Little et al., 2007). Within the domain of the aesthetics of architecture and urban space, a more differential picture of the importance of symmetry has been shown. Investigating the link between the visual, spatial properties of complex streetscapes and their aesthetic judgments, Weber et al. (2008) found that visual regularity informed by parameters of symmetry and uniform arrangements of lateral boundaries in the streetscape photographs seems to be a primary factor in beauty preferences. Though, analyses controlling for professionalism in architecture and urban planning, indicated that symmetry/asymmetry seem to be differentially important factors for beholders of different expertise levels.

Along with symmetry, the complexity factor of an aesthetic stimulus has been discussed as a strong predictor of aesthetic judgment (Jacobsen and Höfel, 2001). The effects of both factors on aesthetic judgment seem to be quite robust, even across different stimuli, participants and contexts (Tinio and Leder, 2009). Interestingly, more recent accounts investigating familiarization with stimuli have revealed findings that are inconsistent with study results on mere-exposure (e.g., Zajonc, 1968). Thus, the robust effects of symmetry and complexity seem to be modifiable by massive familiarization (Tinio and Leder, 2009), which has been thought of eliciting a subsequent preference of novel stimuli (Biederman and Vessel, 2006).

A recent comparative eye-tracking study across cultures and species showed that humans with a different cultural background (Namibian hunter-gatherers and German town dwellers) both showed longer fixation on symmetric abstract graphic stimuli (adopted from Jacobsen and Höfel, 2002), irrespective of pattern complexity, whereas non-human primates (orangutans) did not differentiate between symmetric and non-symmetric patterns but showed faster and broader scanning of the presented screen (Mühlenbeck et al., 2016). Comparison of aesthetic evaluations between both human groups revealed that aesthetic preferences were in accordance with fixation preference only within the German subject group, but not in the Namibian group.

One of the strongest contenders for a universal law of beauty has been considered the Golden Section. In "Vorschule der Ästhetik," Fechner (1876) illustrated the role of the Golden Section Hypothesis in determining aesthetic preferences. Importantly, there are empirical accounts both supporting and contradicting (for an overview, see e.g., Konečni, 2012) the idea of the fundamentally important golden ratio, the latter accounts pointing at a high susceptibility of the effect to studies' methodological differences (Höge, 1995).

Another theoretical aspect which has been thought of as constituting a universal is the correspondence of basic colors and forms. If such a correspondence exists, we would expect it to be highly stable. The desire to find basic, generally applicable principles for art and design in early 20th century gave rise to theorizing about a universal and ideal visual language, and resulted in the color-form assignments developed by Wassily Kandinsky (Jacobsen, 2002, 2004b). Thenceforward Kandinsky's assigned yellow triangle, red square and blue circle became a well-known theme in the Bauhaus school of design. Remarkably, assessing color-form assignments in those a study with nonartist students, Jacobsen (2002) could show that Kandinsky's combinations were the least preferred. In both tasks (mere color-form correspondence vs. aesthetic correspondence), about half of the participants showed clear and stable assignments (red to triangle, blue to square and yellow to circle) and stated world knowledge associations in the rationale for their choice. Yet, another study with visual arts experts demonstrated different color-form assignments (Jacobsen and Wolsdorff, 2007). Those results led to the notion that a multitude of factors (i.e., world knowledge, education, and historical change, as well as individual, group-specific, and societal leitmotifs) influence individual color-form preferences and that the Bauhaus combinations were historically/culturally determined (Jacobsen and Wolsdorff, 2007).

Another combination of basic perceptual qualities such as color and sound and their corresponding approach-avoidance patterns have been investigated recently in a comparative eyetracking study on human and non-human primate species (Mühlenbeck et al., 2015). Regarding biases for and avoidance of colors in human subjects, this study only partially supported previous findings (i.e., avoidance of yellow). Eye-tracking data for non-human primates (orangutans) did not indicate clear gaze duration biases or avoidance for color at all. Other than hypothesized, no modulation of color approach-avoidance patterns by consonant and dissonant auditory stimuli could be found for both human and non-human subjects. The authors concluded that in specific contexts color preferences could be independent of perceiving auditory stimuli and that the lack of specific color biases and color avoidances in both subject groups neither support nor disagree with hypotheses regarding the advantageous evolution of trichromatic color vision.

Among all of the contenders for aesthetic universals one of the most realized across cultures seems to be the factor of skin quality. Apart from a few exceptional examples regarding beauty related skin condition (e.g., decorative scarring in African tribes, facial tattooing in women of Myanmar's chin tribe), a flawless and even skin texture as well as homogeneity of skin color seem to be primary determinants of perceived attractiveness (e.g., Symons, 1995; Fink et al., 2001, 2006). This influence of skin quality has been explained in terms of perceived higher health condition, reproductive ability and youth in general. 


\section{Visual Art and Photography}

Research on aesthetic preference in visual arts has so far focused on group differences in order to find general rules. However, individual differences of aesthetic judgments have been revealed in few different domains, one of them being that of visual art (e.g., Jacobsen and Höfel, 2002; Jacobsen, 2004a).

Höfel and Jacobsen (2003) could demonstrate that beauty judgments of novel, formal graphic patterns (taken from Jacobsen and Höfel, 2002) made by undergraduate students with no expert knowledge in fine arts were changing within several weeks, thereby showing low stability of individual judgments of aesthetic value. Following up on this finding, Jacobsen (2004a) investigated individual differences in beauty judgments of another set of novel, formal graphic patterns, comparing individual performance and group models for ratings and rankings made by a homogeneous group of non-artist college students. It was found that the group model, based on data averages, could only account for half of the participants' judgments, whereas better representation of the data was achieved by the individual models. Beauty judgments of a number of participants even resulted in contrary ratings and rankings, being intra-individual consistent.

In those studies, which used novel graphic patterns, the individual differences in aesthetic judgment of beauty would have led to null results if prescriptively classified objects would have been grouped for the analysis. Rather, individual judgment policies have been captured using methods of judgment analysis. This way, different subsets of stimuli have contributed to conditions in different participants. In view of the above findings, which indicated manifest individual differences in aesthetic preferences, it may be essential to group judgment categories rather than object categories. As implemented by Jacobsen et al. (2006), identifying brain correlates of aesthetic judgments of beauty using these graphic patterns was only possible by employing this approach.

Few studies have also shown individual differences for color preferences (McManus et al., 1981; Whitfield, 1984). Those preferences appear to depend to a great extent on the context, specifically on the object to be colored. Regarding the study by Whitfield (1984), participants showed substantial individual differences of color preferences for walls of a domestic interior furnished in one of three styles (Modern, Georgian, Art Nouveau) when ask to perform corresponding appropriateness rankings of given color samples.

Since at least conceptual art emerged in the middle of the 20th century, it stands to reason that defining art requires reference to its context (Gartus et al., 2015). Contextual influences on emotional responses toward different kinds of artworks (abstract paintings vs. graffiti artworks) were demonstrated in a study by Gartus and Leder (2014). They presented both types of artworks in one of two contexts (museum vs. natural street scene) and showed that participants with a positive attitude toward graffiti art even elicited more positive evaluations of those artworks in a street context (compared to a museum context). The authors concluded that individual attitudes toward art styles modulated contextual effects on the evaluation of artworks. Following up on those results, Gartus et al. (2015) investigated specifically which effects context could have on perception and appreciation of an artwork. Again they found that aesthetic appreciation can be significantly influenced by an artworks context and that some of those effects depend on the style of artworks and some result from an interaction of context and individual interest in art styles.

In another study, implicit aesthetic preferences for figurative as opposed to abstract art styles have been shown (Mastandrea et al., 2011), involving shorter response latencies when participants (non-experts in arts) associated positive (vs. negative) words with figurative (vs. abstract) art.

As to the aesthetics of photography, to date few studies have investigated specific attributes that might account for favoring one (artistic) photograph over another. Often photographs have been used as a technical means to investigate other questions within experimental aesthetics and most experimental approaches, directed at photography itself, focused more on the perceptual than on aesthetic processing of photographs. Certainly, some of the attributes which are discussed as being relevant for aesthetic preference in visual and fine arts might be relevant for the aesthetics of photographs as well. The few empirical findings suggest that features determining image quality seem to be important for the liking of a photograph. In two studies, Tinio and Leder (2009) and Tinio et al. (2011) used images of natural and human-made scenes for which they manipulated certain image features (systematic degradations of contrast, grain size, and sharpness/focus). Depending on the type of scene (stronger effects in human-made as compared to natural scenes), they found that certain degradations and their combinations (higher contrast, less grain, better focus) had more impact on aesthetic preference than others. Furthermore, their results showed an additive effect for feature degradations (an image was less liked the more degradations it was subjected to).

Composition plays a role in a lot of stimuli that can be experienced aesthetically. To examine compositional effects experimentally, a few studies focused for instance on cropping of photographs and its determinants (McManus et al., 2011; Abeln et al., 2016). It was observed, that cropping ability differed substantially between individuals and that cropping is driven more by top-down knowledge of objects (McManus et al., 2011) and that saliency and balancing of different salience regions seems to play an important role for aesthetic appreciation and preference (Abeln et al., 2016). Regarding image attributes that are traditionally discussed within visual aesthetics, such as the rule of thirds and the golden section, the rare studies investigating those in the context of photographs did not reveal a clear pattern of findings yet. Surprisingly a lot of factors which may influence the aesthetic appreciation of photographs have not been or have been only insufficiently investigated yet. To the best of our knowledge, this applies as well to the development of taste in art photography.

\section{Music}

Up to date neuro-cognitive models of human mental facilities and sensory modalities are quite sophisticated. In view of substantial differences in the mental processing architecture across domains like vision and audition, it stands to reason that there are domain-specific characteristics of music processing which have 
to be taken into account in addition to modality- and domainindependent processing stages. This has been implemented in the neuro-cognitive model proposed by Brattico et al. (2013), in which domain-specificity is considered an important aspect in the aesthetic processing of music.

In contrast to other aesthetic domains like dance, language, or architecture, research on the aesthetic appreciation in the musical content domain has been progressed notably within the last decade. The respective model by Brattico et al. (2013) now constitutes a relevant reference point for present-day research. According to their elaborations, initial processing stages (feature analysis, multisensory integration, top-down cognitive processing) in the course of an aesthetic appreciation of a musical stimulus, are common among individuals which share the same background of musical culture. Subsequent stages are characterized by controlled cross-modal neural processes underlying intermingled cognitive, affective, and decisional processes which result in aesthetic emotions, aesthetic judgments and conscious liking. Although often used as synonyms, the authors differentiate "liking", which is considered as a more ongoing process, from "preferences." Preference for a musical style or genre is regarded as stable over time and has been shown to be determined for instance by social factors (peer groups, musical taste of parents and siblings, especially during adolescence; Roulston, 2006) and an individual's personality (e.g., Rentfrow and Gosling, 2003; North, 2010). Research on musical genre preferences have to take into account difficulties in defining genres and the respective interindividual differences as well as the development of genres and of their sociocultural meaning over time, which might limit the validity of assessed genre preferences. Other than for musical genres, preferences for a musical instrument seem to be more context-sensitive, at least in children, whose choices of musical instruments have been shown to be susceptible to social schemata such as gender stereotypes (Harrison and O'Neill, 2000).

Research examining those factors that are assumed to account for a certain variability of musical liking has focused, e.g., on mood (for a review on general mood influences, see Konečni, 2010; on sad music and mood congruency, see Sachs et al., 2015), attention and intentionality (for an overview, see Brattico et al., 2013). As summarized by Brattico et al. (2013), a certain variability of the aesthetic experience of music is brought about by internal (e.g., expertise, mood, attitudes) and external context factors (environment, peers, task at hand). One explanation for an influence of expertise on musical preferences (as shown, e.g., by Müller et al., 2010; Brattico et al., 2016) seems to be that expertise provides specific prototypes (Leder et al., 2004). Prototypicality has been proposed to be preferred (Martindale, 1988) and for the domain of music it has been shown to positively influence preferences (Smith and Melara, 1990; Brattico et al., 2009b).

Another interesting study showed that lyrics (as another external factor) have differential effects in aesthetic experience of happy and sad music, that is instrumental cues were shown to be more important in happy music (with stronger positive emotions for happy music without lyrics), both happy and sad musical pieces without lyrics were liked more than those with lyrics, and lyrical cues seemed to be more important for defining and inducing sad emotions (Brattico et al., 2011). Attribution of a value (positive or negative) to music is observable during an individual's life span and across cultures (Brattico et al., 2009a). On a neural level, basic emotion perception was associated with structures being spatially separate from motivational and evaluative processes and seems to be processed earlier than the latter (Brattico et al., 2016). This implicit appraisal of the affective state which is induced by a musical piece seems to build the basis for aesthetic enjoyment, conscious liking and various judgments linked to the aesthetic episodes (e.g., beauty judgment; Brattico et al., 2016). Sound features like complexity or sensory dissonance can be linked to more stable aspects of aesthetic experiences and to domain general perceptual preferences across different musical and auditory domains (Brattico et al., 2009a). More specifically temporally or spectrally complexity is preferred over less complexity (Tervaniemi et al., 2000; Brattico et al., 2001; Fujioka et al., 2004) whereas sensory dissonance is shown to be related to avoidance behavior of individuals as early as 2 months old (Trainor et al., 2002). It has been proposed that the listening context (everyday situation vs. aesthetic context like a concert hall) might either induce solely basic emotions (everyday situations) or special aesthetic emotions such as aesthetic enjoyment, awe, nostalgia or being moved (Brattico and Pearce, 2013).

The numerous factors and their netting of complex interactions, which are thought to influence the aesthetic experience of music, seem to account for a high variance of subjective musical preferences (Brattico and Jacobsen, 2009).

\section{Language and Literature}

A recent study shed light on the conceptual structure underlying the aesthetics of literature, using a verbal association task (Knoop et al., 2016). The conceptual representations were also investigated specifically for the aesthetics of literary forms and genres (short stories, novels, poems, comedies, plays). Comparison to findings for other conceptual structures in the domains of visual art and music revealed the greatest overlap between literature and music. Particularly, as beauty was named as one of the most frequent terms for almost all domains investigated, it seems to be an important category for nonexpert conceptualization of aesthetics (Jacobsen et al., 2004; Istók et al., 2009; Augustin et al., 2012; Knoop et al., 2016). Though, Knoop et al. (2016) derived from their findings a different role of the term beautiful when literature is denoted. More specific, rather prosodic language qualities and feelings (or moods or atmospheres) described as romantic or poetic seem to underlie an attribution of literary texts as beautiful. For overall aesthetics of literature, suspenseful was another prevalent term. Apart from that, the most frequent labels varied between literary subgenres. In addition, there was a predominance of evaluative (compared to few descriptive) terms, as previously found for the aesthetics of objects (Jacobsen et al., 2004). Knoop et al. (2016) suggested that within aesthetic conceptualization even the apparently purely descriptive labels may comprise an evaluative dimension. For instance, rhythmic was among their top listed music-related terms for poetry (besides harmonious and melodious) and in a 
previous study regularity in poems was associated with positive aesthetic liking judgments (Obermeier et al., 2013). There are also empirical accounts that indicate that even when not explicitly instructed to do so, spontaneous evaluation takes place during silent reading (Bohrn et al., 2013).

It was discussed, that it was not possible to derive to which extent the assessed conceptual representations for the aesthetics of literature (and subgenres) arose by reason of acquired theoretical concepts or by the participant's own experience with the contemplated subgenres (Knoop et al., 2016). Thus, although there are rare empirical endeavors yet which investigated literary aesthetic preferences, their degree of stability and potential influencing factors accounting for a certain context-dependent variability, the presented information regarding the concepts associated with aesthetic attribution in the domain of literature contribute to a deeper understanding of how aesthetic appeal dimensions are perceived and conceptualized across aesthetic domains.

\section{Dance}

It has been suggested that watching a dance movement involves a simulation of this movement using the same neural network that is active while executing it (Calvo-Merino et al., 2005). It might be reasonable or even essential to include considerations of more basic movements in order to tackle the aesthetics of dance. Orgs et al. (2013) have broken down the aesthetic experience into the investigation of aesthetic effects of three levels of human movement representations. Their findings indicate that aesthetic preferences can be influenced by body postures, movement continuation ("good" vs. "bad") and choreographic structure. The finding, that initial familiarization with asymmetrical sequences increased later liking of such asymmetrical sequences, also suggest a structural mere exposure effect.

Drawing upon the discovery of mirror neurons and the concept of embodied simulation, it has been argued that the activation of universal mechanisms encompassing the simulation of emotions, actions and bodily sensations, is a crucial part of the observer's aesthetic response to those stimuli (Freedberg and Gallese, 2007). According to them, such embodied resonance in response to an image can also arise if emotions, actions and bodily sensations are merely implied by the physical traces of artwork production and even in the absence of an overt emotional component. Cross et al. (2011) suggested that the above conceptualization could be further applied to more dynamic forms of art and they reasoned that embodied simulation may influence the aesthetic experience of dance performances. In their fMRI study, they had participants judge how well they think they were physically able to reproduce a range of dance movements (performed by professional ballet dancers) and how much they liked those movements. Results indicated a preference for watching more difficult movements (associated with a higher activation of bilateral occipitotemporal and right inferior parietal portions of the action observation network (AON)). It seems that increased aesthetic ratings for dance movements that have been mentally simulated or even physically practiced do not result from mere exposure or familiarity effects, as suggested by findings of later training studies (Kirsch et al., 2013, 2015).
The findings by Kirsch et al. (2013) didn't show increased enjoyment of dance movements in the view and music, or musiconly conditions, suggesting that actual performance of dance movements additionally contributes to the aesthetic experience of dance. Cross (2015) described the interaction of embodiment and aesthetic experience as resembling a $\mathrm{U}$-shaped function. This perspective might partially explain the high preferences for familiar and likely physically reproducible dance movements as well as for those dance movements which are spectacular, novel and not physically reproducible.

In their studies Stevens et al. (2009) used personal digital assistants (PDAs) to assess psychophysiological data of participants viewing a dance performance. In contrast to emotional arousal, which has been higher during more important dance parts (according to choreographer and experimenter ratings), no correlations with specific dance parts have been found for emotional valence. A wide variety of individual's interpretation of and experience with dance performances has been discussed as a potential factor that led to the latter result.

\section{Architecture and Environmental/Urban Space}

Consideration of aesthetic aspects is part of architectural creation itself, thus both are closely linked and, perhaps to a similar extent like in the domain of visual arts, widely discussed within philosophical, architectural, and psychological frames. Research endeavors in the field have tried to tackle general principles for an influence of physical features on the evaluation of aesthetic experience of buildings and their environments (Nasar, 1994). As reviewed by Nasar (1994), three kinds of aesthetic variables became apparent on that score, that is formal variables (factors such as complexity, enclosure and order), symbolic variables (e.g., style) and schema (e.g., typicality). The author's analyses of empirical accounts derive advice for design reviewers seeking different evaluative responses based on combinations of the above listed feature characteristics. Besides considerations of aesthetically relevant aspects of buildings themselves, features of a building's context, ranging from closely outlined surroundings to vast streetscapes encompassing several other objects and their features within urban space (e.g., parks, stations, railways, space between objects), are also regarded in terms of their influence on aesthetic appreciation and should be addressed just as well within experimental aesthetics. Selecting habitats and creating shelter are arguably very old human behavior.

First, research findings fitting the more narrowly drafted architectural domain will be reviewed before addressing the aesthetics of urban space in the second part of this paragraph.

Gifford et al. (2000) had architects and laypersons aesthetically judge their global assessment (ranging on a 10-point scale from terrible to excellent architecture) of modern office buildings. Additionally, assessed pleasure and arousal ratings for those building photographs have been related to objective features of the buildings. Global assessments of the buildings have been found to be based on elicited pleasure but not on elicited arousal for both groups. The authors found, that aesthetic evaluations of experts and laypersons were almost unrelated and they concluded that this might be due to the fact, that both groups based their 
emotional assessments on different groups of objective features of the building's exterior. For expert participants pleasure regarding an office building has been related to objective features such as more metal cladding, more railing, and fewer arches. Those physical features seemed to signify more complex ideas for architects, such as prototypicality and richness of material. In a different way, layperson's pleasure ratings were not derived from even one of the assessed objective building features. This has been explained in the context of layperson's greater within-group heterogeneity of pleasure ratings, the latter being more subject to various influences in laypersons in contrast to expert ratings of architecture. However, an additional interesting result has been, that none of the three assessed contextual variables (visible amount of landscaping and roads, human presence/activity evidence) had a significant influence on global assessments, pleasure, and arousal ratings, which indicated little impact of non-building context.

Another study by Marković and Alfirević (2015) compared artist's and non-artist's aesthetic judgments of architectural expressiveness of photographed buildings. Two clusters of architectural objects have been obtained within analyses. A choleric expressiveness cluster including objects rated high on the factors aggressiveness and color and low on the factor regularity, and the phlegmatic expressiveness cluster encompassing architectural examples with low ratings on Aggressiveness and Color, and high ratings on Regularity. The authors associated objects of the former choleric cluster with expressionistic and the latter phlegmatic cluster objects with minimalist architectural character. Interestingly, further analyses suggested that although the two groups of architectural objects differed regarding their allocated expressiveness, aesthetic pleasure has been rated virtually equal for both across participants. Expertise related differences in aesthetic preferences (experts rated objects included in the phlegmatic cluster as more aesthetic, whereas non-experts judged choleric cluster objects as more aesthetic) have been interpreted in terms of processing fluency and Berlyne's (1971) optimal level of arousal. Marković and Alfirević referred to architects (meaning experts, as against non-experts) as being more responsive to new experiences and as preferring abstract and less comprehensible content (compared to non-experts' preferences of clearer and pleasant content; empirical accounts by Rawlings, 2000, 2003). Given that, they argued that the phlegmatic cluster might have encompassed objects that have been experienced as more interesting and thus more pleasant for experts, whereas the same objects seemed to have been incomprehensible (less fluently processable), boring and thus less pleasant for non-experts. The latter experienced the presented architectural objects as more aggressive (compared to experts), which has been interpreted as representing higher negative arousal resulting from a lack of competence and safety feeling. Linking those considerations with the results found by Gifford et al. (2000), it is interesting, that the latter found no relation between arousal ratings and general assessments of architectural quality and pleasure experience.

Familiarity with, prototypicality and simplicity of architectural objects as influencing factors on automatic aesthetic evaluation (via effecting processing fluency) have been discussed as well by Mastandrea et al. (2011). Using the IAT, they observed shorter reaction times when non-expert participants were asked to associate positive words with classical architecture (compared to longer reaction times when associating with contemporary architecture). This response pattern corresponded with the one assessed for explicit evaluations for both architectural styles. Note that this correlation has not been found for the other stimulus type, i.e., figurative and abstract artworks. The authors concluded that, on the individual level, participants might have shown the same evaluative trend in both implicit and explicit aesthetic evaluation tasks because variety of stimuli within each architectural category is relatively low (which is not the case regarding art categories).

One physical feature which has always been regarded as important for architectural planning and designing (Le Corbusier, 1927) and which has been shown to influence emotional reactions (e.g., Hevner, 1935) and aesthetic preferences (e.g., Leder and Carbon, 2005; Bar and Neta, 2006) is contour, especially its amount of curvature. In one of the first fMRI studies on the influence of physical features of the built environment on brain activation, Vartanian et al. (2013) demonstrated that the effect of contour on beauty judgments can be extended to architecture. In their study non-expert participants showed beauty preferences for curvilinear over rectilinear spaces and on behavioral level this effect has been shown to be likely driven by pleasantness. Moreover, contemplating beauty of curvilinear spaces was associated exclusively with increased activity of the anterior cingulate cortex (ACC) over beauty judgments of rectilinear spaces. It has been demonstrated that the ACC contributes to reward and emotional processing (Kringelbach and Rolls, 2004; Liu et al., 2011). Taking together behavioral and neural results, Vartanian et al. (2013) conclude that aesthetic preferences for curvilinear contour is underpinned by reward and emotion, once again pointing to the role of emotion in aesthetic experience.

In this 2013 study, stimuli were controlled for the variables height and openness/enclosure because of some empirical results hinting at their influence on cognition and emotion when contemplating architecture (Franz et al., 2005; Meyers-Levy and Zhu, 2007). In a later study, the authors investigated the effects of those variables on aesthetic judgments, again by assessing behavioral and fMRI-data (Vartanian et al., 2015). Their results showed that higher ceilings and open rooms were more likely to be judged as more beautiful. Increased activity in frontal and parietal structures of the dorsal stream associated with visuospatial exploration and attention have been demonstrated for higher ceilings. Moreover, the authors suggested that involvement of the temporal lobes when judging the beauty of open rooms might be linked to their contribution to the temporal dynamics of visual motion representation.

Having focused on the aesthetics of buildings and their interior so far, it remains to address the influence of the context in which those buildings are beheld. With regard to the aesthetics of urban landscapes, it has been shown that urban scenes that constitute places with panoramic views, settings with historical/cultural significance and recreational places were considered as most 
attractive, whereas industrial and housing areas were rated as most unattractive (Galindo and Hidalgo, 2005). However, those categorical results were derived from listings of the most and least attractive places of one city (Málaga, Spain). The participants, who were residents who indicated a high sense of belonging for the city, also evaluated the restorative properties and the environmental characteristics of their listed places. The authors based the formation of the above listed urban aesthetic categories on two dimensions, that is the function and historical value of a scene, which they consider as important influencing factors regarding urban scene preferences. Findings by Van den Berg et al. (2003) and Galindo and Hidalgo (2005) further support the idea that the restorative capacity of a scene seems to be one of the criteria underlying environmental aesthetic preferences (Purcell et al., 2001).

Even more interesting than assessing preferences for rather broad urban aesthetic scenes is the question, to which visual, spatial properties those aesthetic preferences can be attributed. Weber et al. (2008) were narrowing down this issue by assessing the relationship between aesthetic judgments and selected visuospatial properties of complex streetscapes sampled from an existing mid-sized German town. Vegetation, stylistic uniformity, homogeneity of scale, and symmetry were observed as important factors. Corresponding to Gestalt psychology's figure formation characteristics (later adapted for 3D architectural spaces by Weber, 1995) the results of participants' beauty ratings suggested visual regularity as primary factor in beauty judgment. Precisely, factors such as symmetry and uniformity of lateral spatial boundaries of the streetscapes depicted in the photographs were influencing aesthetic judgments especially if those boundaries were formed by vegetation or by building types featuring stylistic uniformity. Surprisingly, the study revealed no significant differences between aesthetic judgments of experts and laymen.

\section{Design}

Nowadays a lot of entities can be "designed" in some way. In this section, we will shortly look at the few research findings addressing the more traditional fields of design. Empirical accounts indicate that, regarding the aesthetic appreciation of objects, usability of those objects or products is positively affected by visual aesthetics, but that this relation is mediated by an overall evaluation of "goodness" of the respective object (for a review, see e.g., Hassenzahl and Monk, 2010). Individual differences of aesthetic preferences for wall color of a domestic interior, which was furnished in one of three styles, was found by Whitfield (1984). An examination of the preferences for pieces of furniture, which should be also categorized according to those three styles, showed once more, that prototypical or familiar objects were preferred (Whitfield and Slatter, 1979). An influence of attitudes on interior design preferences has been demonstrated by Ritterfeld (2002). However, this effect has been solely shown to arise from social heuristic processing when the social information of the object (a piece of furniture) was decodable or consistent. This way of processing is thought to be more automatic and less controlled. In contrast, systematic analysis of the object's structural, non-social properties appeared when social information was not decodable or inconsistent. Thus, failure of the social heuristic resulted in an increase of preference judgment latencies and a decrease of evaluation certainty. Even so, taking into account motivational factors, an automatic application of social heuristic processing can be overcome when accuracy or directional goals are present. To explain everyday aesthetic experiences and preferences, Ritterfeld (2002) proposed an integrative model comprising both target properties and motivational aspects as determining factors. Another study that also used chairs (classic vs. modern) as target design objects, investigated whether expertise in industrial design would influence preferences for the different styles on an implicit and explicit level (Mastandrea and Maricchiolo, 2014). The results indicated an automatic influence of expertise on aesthetic evaluation of those objects, with laypeople showing no specific preference orientation compared to experts who preferred modern over classic chairs.

\section{CONCLUSION}

We have examined aspects of variability and stability regarding the aesthetic experience and aesthetic preferences for different domains of aesthetic appreciation. Within this scope, we wanted to rivet on the role of sensitive periods for the development of aesthetic taste, as well as on the importance of cognitive schemata and specifically scripts for the aesthetic experience in various aesthetic domains.

Bearing in mind that all aesthetic experience is situated and processed by individuals whose ontological development was influenced by nature and nurture (e.g., Jacobsen, 2006, 2010a), the diverse empirical pattern obtained in the present analysis may not be surprising. People differ and aesthetic domains do as well. Although some preferred features appear to be deeply rooted in the visual modality, symmetry or green vegetation, the visual aesthetic domains appear to show the highest variability. While mixed accounts of variability and stability were found for the domains of music, language, and architecture. It is important to note, however, that the mosaic of available research is patchy. While notions on sensitive periods in the development of musical taste exist, comparable research for visual domains of aesthetic appreciation is scarce. In the same vein, comparisons of individual differences of aesthetic experience across domains are currently hardly possible.

We suggest that within ontogenesis of the human perceptual and conceptual system, crucial foundations for the capacity of aesthetic evaluation are laid. To date, research on sensitive (or even critical) periods regarding, for instance, the development of basic characteristics of human sensory processing as well as of higher-order processes relevant for aesthetic appreciation, is still somewhat limited. In order to link such relevant sensitive periods to periods which may be crucial for the development of aesthetic taste, further research is required. Therefore, it will be important to isolate even more those perceptual and cognitive processes (and relevant interactions) which play a crucial role for the formation of aesthetic evaluations. We also believe that calling some attention to the significance of cognitive schemata 
and scripts in experimental aesthetics may help to understand some empirical findings better and add to models of aesthetic processing in various domains.

\section{REFERENCES}

Abeln, J., Fresz, L., Amirshahi, S. A., McManus, I. C., Koch, M., Kreysa, H., et al. (2016). Preference for well-balanced saliency in details cropped from photographs. Front. Hum. Neurosci. 9:704. doi: 10.3389/fnhum.2015.00704

Ahern, A., Bennett, K. M., and Hetherington, M. (2008). Internalization of the ultra-thin ideal: positive implicit associations with underweight fashion models are associated with drive for thinness in young women. Eat. Disord. 16, 294-307. doi: 10.1080/10640260802115852

Augustin, M. D., Wagemans, J., and Carbon, C.-C. (2012). All is beautiful? Generality vs. specificity of word usage in visual aesthetics. Acta Psychol. (Amst.) 139, 187-201. doi: 10.1016/j.actpsy.2011.10.004

Bar, M., and Neta, M. (2006). Humans prefer curved visual objects. Psychol. Sci. 17, 645-648. doi: 10.1111/j.1467-9280.2006.01759.x

Bartlett, F. C. (1932). Remembering: A Study in Experimental and Social Psychology. Cambridge: Cambridge University Press.

Berlyne, D. E. (1971). Aesthetics and Psychobiology. New York, NY: Appleton Century-Crofts.

Biederman, I., and Vessel, E. A. (2006). Perceptual pleasure and the brain. Am. Sci. 94, 249-255. doi: 10.1511/2006.3.247

Bohrn, I. C., Altmann, U., Lubrich, O., Menninghaus, W., and Jacobs, A. M. (2013). When we like what we know - A parametric fMRI analysis of beauty and familiarity. Brain Lang. 124, 1-8. doi: 10.1016/j.bandl.2012.10.003

Brattico, E., Alluri, V., Bogert, B., Jacobsen, T., Vartiainen, N., Nieminen, S. K., et al. (2011). A functional MRI study of happy and sad emotions in music with and without lyrics. Front. Psychol. 2:308. doi: 10.3389/fpsyg.2011.00308

Brattico, E., Bogert, B., Alluri, V., Tervaniemi, M., Eerola, T., and Jacobsen, T. (2016). It's sad but I like it: the neural dissociation between musical emotions and liking in experts and laypersons. Front. Hum. Neurosci. 9:676. doi: 10.3389/ fnhum.2015.00676

Brattico, E., Bogert, B., and Jacobsen, T. (2013). Toward a neural chronometry for the aesthetic experience of music. Front. Psychol. 4:206. doi: 10.3389/fpsyg.2013. 00206

Brattico, E., Brattico, P., and Jacobsen, T. (2009a). The origins of the aesthetic enjoyment of music - a review of the literature. Music. Sci. 13, 15-39. doi: $10.1177 / 1029864909013002031$

Brattico, E., and Jacobsen, T. (2009). Subjective appraisal of music: neuroimaging evidence. Ann. N. Y. Acad. Sci. 1169, 308-317. doi: 10.1111/j.1749-6632.2009. 04843.x

Brattico, E., Jacobsen, T., De Baene, W., Glerean, E., and Tervaniemi, M. (2010). Cognitive vs. affective listening modes and judgments of music - An ERP study. Biol. Psychol. 85, 393-409. doi: 10.1016/j.biopsycho.2010.08.014

Brattico, E., Näätänen, R., and Tervaniemi, M. (2001). Context effects on pitch perception in musicians and non-musicians: Evidence from ERP recordings. Music Percept. 19, 1-24. doi: 10.1525/mp.2001.19.2.199

Brattico, E., Pallesen, K. J., Varyagina, O., Bailey, C., Anourova, I., Järvenpää, M., et al. (2009b). Neural discrimination of nonprototypical chords in music experts and laymen: an MEG study. J. Cogn. Neurosci. 21, 2230-2244. doi: 10.1162/jocn.2008.21144

Brattico, E., and Pearce, M. (2013). The neuroaesthetics of music. Psychol. Aesthet. Creat. Arts 7, 48-61. doi: 10.1037/a0031624

Brewer, W. F., and Nakamura, G. V. (1984). "The nature and functions of schemas," in Handbook of Social Cognition, Vol. 1, eds R. S. Wyer Jr. and T. K. Srull (Hillsdale, NJ: Erlbaum), 119-160.

Calvo-Merino, B., Glaser, D. E., Grèzes, J., Passingham, R. E., and Haggard, P. (2005). Action observation and acquired motor skills: an FMRI study with expert dancers. Cereb. Cortex 15, 1243-1249. doi: 10.1093/cercor/bhi007

Cross, E. S. (2015). "Beautiful embodiment: the shaping of aesthetic preference by personal experience," in Art, Aesthetics and the Brain, eds M. Nadal, J. P. Huston, L. Agnati, F. Mora, and C. J. Cela-Conde (Oxford: Oxford University Press), 189-208.

\section{AUTHOR CONTRIBUTIONS}

\author{
TJ and SB conceived and wrote the manuscript.
}

Cross, E. S., Kirsch, L., Ticini, L., and Schütz-Bosbach, S. (2011). The impact of aesthetic evaluation and physical ability on dance perception. Front. Hum. Neurosci. 5:102. doi: 10.3389/fnhum.2011.00102

Dobzhansky, T. (1962). Mankind Evolving: The Evolution of the Human Species. New Haven, CT: Yale University Press.

Fazio, R. H., and Olson, M. A. (2003). Implicit measures in social cognition research: Their meaning and use. Annu. Rev. Psychol. 54, 297-327. doi: 10.1146/ annurev.psych.54.101601.145225

Fechner, G. T. (1876). Vorschule der Aesthetik [Experimental Aesthetics; "Preschool" of aesthetics]. Leipzig: Breitkopf and Härtel.

Fink, B., Grammer, K., and Matts, P. J. (2006). Visible skin color distribution plays a role in the perception of age, attractiveness, and health in female faces. Evol. Hum. Behav. 27, 433-442. doi: 10.1016/j.evolhumbehav.2006.08.007

Fink, B., Grammer, K., and Thornhill, R. (2001). Human (Homo sapiens) facial attractiveness in relation to skin texture and color. J. Comp. Psychol. 115, 92-99. doi: 10.1037/0735-7036.115.1.92

Franz, G., von der Heyde, M., and Bülthoff, H. H. (2005). An empirical approach to the experience of architectural space in virtual reality-Exploring relations between features and affective appraisals of rectangular indoor spaces. Autom. Construct. 14, 165-172. doi: 10.1016/j.autcon.2004.07.009

Freedberg, D., and Gallese, V. (2007). Motion, emotion and empathy in esthetic experience. Trends Cogn. Sci. 11, 197-203. doi: 10.1016/j.tics.2007.02.003

Fuhrmann, D., Knoll, L. J., and Blakemore, S. (2015). Adolescence as a sensitive period of brain development. Trends Cogn. Sci. 19, 558-566. doi: 10.1016/j.tics. 2015.07.008

Fujioka, T., Trainor, L. J., Ross, B., Kakigi, R., and Pantev, C. (2004). Musical training enhances automatic encoding of melodic contour and interval structure. J. Cogn. Neurosci. 16, 1010-1021. doi: 10.1162/0898929041502706

Galindo, M. P., and Hidalgo, M. A. (2005). Aesthetic preferences and the attribution of meaning: environmental categorization processes in the evaluation of urban scenes. Int. J. Psychol. 40, 19-26. doi: 10.1080/ 00207590444000104

Gartus, A., Klemer, N., and Leder, H. (2015). The effects of visual context and individual differences on perception and evaluation of modern art and graffiti art. Acta Psychol. (Amst) 156, 64-76. doi: 10.1016/j.actpsy.2015.01.005

Gartus, A., and Leder, H. (2014). The white cube of the cuseum versus the grey cubes of the street: the role of context in aesthetic judgments. Psychol. Aesthet. Creat. Arts 8, 311-320. doi: 10.1037/a0036847

Gifford, R., Hine, D. W., Muller-Clemm, W., Reynolds, D., and Shaw, K. (2000). Decoding modern architecture - A lens model approach for understanding the aesthetic differences of architects and laypersons. Environ. Behav. 32, 163-187. doi: 10.1177/00139160021972487

Hanich, J., Wagner, V., Shah, M., Jacobsen, T., and Menninghaus, W. (2014). Why we like to watch sad films. The pleasure of being moved in aesthetic experiences. Psychol. Aesthet. Creat. Arts 8, 130-143. doi: 10.1037/a0035690

Hargreaves, D. J., and North, A. C. (2010). "Experimental aesthetics and liking for music," in Handbook of Music and Emotion - Theory, Research, Applications, eds P. N. Juslin and J. A. Sloboda (Oxford, NY: Oxford University Press), 515-546.

Harrison, A. C., and O’Neill, S. A. (2000). Children's gender-typed preferences for musical instruments. Music 28, 81-97. doi: 10.1177/0305735600281006

Hassenzahl, M., and Monk, A. (2010). The inference of perceived usability from beauty. Hum. Comput. Interact. 25, 235-260. doi: 10.1080/07370024.2010. 500139

Hevner, K. (1935). Experimental studies of the affective value of colors and lines. J. Appl. Psychol. 19, 385-398. doi: 10.1037/h0055538

Höfel, L., and Jacobsen, T. (2003). Temporal stability and consistency of aesthetic judgments of beauty of formal graphic patterns. Percept. Mot. Skills 96, 30-32. doi: $10.2466 /$ pms.2003.96.1.30

Höfel, L., and Jacobsen, T. (2007a). Electrophysiological indices of processing aesthetics: Spontaneous or intentional processes? Int. J. Psychophysiol. 65, 20-31. doi: 10.1016/j.ijpsycho.2007.02.007 
Höfel, L., and Jacobsen, T. (2007b). Electrophysiological indices of processing symmetry and aesthetics - A result of judgment categorization or judgment report? J. Psychophysiol. 21, 9-21. doi: 10.1027/0269-8803.21.1.9

Höge, H. (1995). Fechner's experimental aesthetics and the golden section hypothesis today. Emp. Stud. Arts 13, 131-148. doi: 10.2190/UHTQ-CFVDCAU2-WY1C

Hooks, B. M., and Chen, C. (2007). Critical periods in the visual system: changing views for a model of experience-dependent plasticity. Neuron 56, 312-326. doi: 10.1016/j.neuron.2007.10.003

Howard-Jones, P. A., Washbrook, E. V., and Meadows, S. (2012). The timing of educational investment: a neuroscientific perspective. Dev. Cogn. Neurosci. 2, 18-29. doi: 10.1016/j.dcn.2011.11.002

Hubel, D. H., and Wiesel, T. N. (1962). Receptive fields, binocular interaction and functional architecture in the cat's visual cortex. J. Physiol. 160, 106-154. doi: 10.1113/jphysiol.1962.sp006837

Istók, E., Brattico, E., Jacobsen, T., Krohn, K., Müller, M., and Tervaniemi, M. (2009). Aesthetic responses to music: a questionnaire study. Music. Sci. 13, 183-206. doi: 10.1177/102986490901300201

Istók, E., Brattico, E., Jacobsen, T., Ritter, A., and Tervaniemi, M. (2013). 'I love Rock ' $n$ ' Roll' - Music genre preference modulates brain responses to music. Biol. Psychol. 92, 142-151. doi: 10.1016/j.biopsycho.2012.11.005

Jacobsen, T. (2002). Kandinsky's questionnaire revisited: fundamental correspondence of basic colors and forms? Percept. Mot. Skills 95, 903-913. doi: $10.2466 /$ pms.2002.95.3.903

Jacobsen, T. (2004a). Individual and group modelling of aesthetic judgment strategies. Br. J. Psychol. 95, 41-56. doi: 10.1348/000712604322779451

Jacobsen, T. (2004b). Kandinsky's color-form correspondence and the Bauhaus Colors: an empirical view. Leonardo 37, 135-136. doi: 10.1162/0024094041139193

Jacobsen, T. (2006). Bridging the arts and sciences: a framework for the psychology of aesthetics. Leonardo 39, 155-162. doi: 10.1162/leon.2006.39.2.155

Jacobsen, T. (2010a). Beauty and the brain: culture, history and individual differences in aesthetic appreciation. J. Anat. 216, 184-191. doi: 10.1111/j.14697580.2009.01164.x

Jacobsen, T. (2010b). "On the psychophysiology of aesthetics: automatic and controlled processes of aesthetic appreciation," in Unconscious Memory Representations in Perception - Processes and Mechanisms in the Brain, eds I. Czigler and I. Winkler (Amsterdam: John Benjamins Publishing Company), 245-257.

Jacobsen, T., Buchta, K., Köhler, M., and Schröger, E. (2004). The primacy of beauty in judging the aesthetics of objects. Psych. Rep. 94, 1253-1260. doi: 10.2466/pr0.94.3c.1253-1260

Jacobsen, T., and Höfel, L. (2001). Aesthetics electrified: an analysis of descriptive symmetry and evaluative aesthetic judgment processes using event-related brain potentials. Emp. Stud. Arts 19, 177-190. doi: 10.2190/P7W1-5F1F-NJK9$\mathrm{X} 05 \mathrm{~B}$

Jacobsen, T., and Höfel, L. (2002). Aesthetic judgments of novel graphic patterns: analyses of individual judgments. Percept. Mot. Skills 95, 755-766. doi: 10.2466/ pms.2002.95.3.755

Jacobsen, T., and Höfel, L. (2003). Descriptive and evaluative judgment processes: behavioral and electrophysiological indices of processing symmetry and aesthetics. Cogn. Affect. Behav. Neurosci. 3, 289-299. doi: 10.3758/CABN.3.4. 289

Jacobsen, T., Schubotz, R. I., Höfel, L., and von Cramon, D. Y. (2006). Brain correlates of aesthetic judgment of beauty. Neuroimage 29, 276-285. doi: 10. 1016/j.neuroimage.2005.07.010

Jacobsen, T., and Wolsdorff, C. (2007). Does history affect aesthetic preference? Kandinsky's teaching of colour-form correspondence, empirical aesthetics, and the Bauhaus. Design J. 10, 16-27. doi: 10.2752/146069207789271902

Kanwisher, N., McDermott, J., and Chun, M. (1997). The fusiform face area: a module in human extrastriate cortex specialized for the perception of faces. J. Neurosci. 17, 4302-4311. doi: 10.3410/f.717989828.793472998

Kanwisher, N., and Yovel, G. (2006). The fusiform face area: a cortical region specialized for the perception of faces. Philos. Trans. R. Soc. Lond. B Biol. Sci. 361, 2109-2128. doi: 10.1098/rstb.2006.1934

Kirsch, L., Drommelschmidt, K., and Cross, E. S. (2013). The impact of sensorimotor experience on affective evaluation of dance. Front. Hum. Neurosci. 7:521. doi: 10.3389/fnhum.2013.00521
Kirsch, L. P., Dawson, K., and Cross, E. S. (2015). Dance experience sculpts aesthetic perception and related brain circuits. Ann. N. Y. Acad. Sci. 1337, 130-139. doi: $10.1111 /$ nyas. 12634

Knoop, C., Menninghaus, W., Wagner, V., and Jacobsen, T. (2016). Mapping the aesthetic space of literature "from below". Poetics 56, 35-49. doi: 10.1016/j. poetic.2016.02.001

Konečni, V. J. (2010). “The influence of affect on music choice," in Handbook of Music and Emotion - Theory, Research, Applications, eds P. N. Juslin and J. A. Sloboda (Oxford, NY: Oxford University Press), 697-723.

Konečni, V. J. (2012). Empirical psycho-aesthetics and her sisters: substantive and methodological issues - Part I. J. Aesthet. Educ. 46, 1-12. doi: 10.5406/jaesteduc. 46.4.0001

Kornysheva, K., von Cramon, D. Y., Jacobsen, T., and Schubotz, R. I. (2010). Tuning-in to the beat: aesthetic appreciation of musical rhythms correlates with a premotor activity boost. Hum. Brain Mapp. 31, 48-64. doi: 10.1002/hbm. 20844

Kral, A. (2013). Auditory critical periods: a review from system's perspective. Neuroscience 247, 117-133. doi: 10.1016/j.neuroscience.2013.05.021

Kringelbach, M. L., and Rolls, E. T. (2004). The functional neuroanatomy of the human orbitofrontal cortex: evidence from neuroimaging and neuropsychology. Prog. Neurobiol. 72, 341-372. doi: 10.1016/j.pneurobio.2004. 03.006

Kuhl, P. K. (2010). Brain mechanisms in early language acquisition. Neuron 67, 713-727. doi: 10.1016/j.neuron.2010.08.038

Le Corbusier (1927). Towards a New Architecture. London: Architectural Press.

LeBlanc, A., Sims, W. L., Silvola, C., and Obert, M. (1996). Music style preferences of different age listeners. J. Res. Music Educ. 44, 49-59. doi: 10.2307/3345413

Leder, H., Belke, B., Oeberst, A., and Augustin, D. (2004). A model of aesthetic appreciation and aesthetic judgments. Br. J. Psychol. 95, 489-508. doi: 10.1348/ 0007126042369811

Leder, H., and Carbon, C.-C. (2005). Dimensions in appreciation of car interior design. Appl. Cognit. Psychol. 19, 603-618. doi: 10.1002/acp.1088

Lewis, T. L., and Maurer, D. (2005). Multiple sensitive periods in human visual development: evidence from visually deprived children. Dev. Psychobiol. 46, 163-183. doi: 10.1002/dev.20055

Little, A. C., Apicella, C. L., and Marlowe, F. W. (2007). Preferences for symmetry in human faces in two cultures: data from the UK and the Hadza, an isolated group of hunter-gatherers. Proc. Biol. Sci. 274, 3113-3117. doi: 10.1098/rspb.2007.0895

Little, A. C., Jones, B. C., and DeBruine, L. M. (2011). Facial attractiveness: evolutionary based research. Philos. Trans. R. Soc. B 366, 1638-1659. doi: 10. 1098/rstb.2010.0404

Liu, X., Hairston, J., Schrier, M., and Fan, J. (2011). Common and distinct networks underlying reward valence and processing stages: a meta-analysis of functional neuroimaging studies. Neurosci. Biobehav. Rev. 35, 1219-1236. doi: 10.1016/j. neubiorev.2010.12.012

Mandler, J. M. (1984). Stories, Scripts, and Scenes: Aspects of Schema Theory. Hillsdale, NJ: Erlbaum, 2-3.

Marković, S., and Alfirević, D. (2015). Basic dimensions of experience of architectural objects' expressiveness: effect of expertise. Psihologija 48, 61-78. doi: 10.2298/PSI1501061M

Martindale, C. (1988). "Aesthetics, psychobiology, and cognition," in The Foundation of Aesthetics, Art and Art Education, eds F. H. Farley and R. W. Neperud (New York, NY: Praeger), 7-42.

Mastandrea, S., Bartoli, G., and Carrus, G. (2011). The automatic aesthetic evaluation of different art and architectural styles. Psychol. Aesthet. Creat. Arts 5, 126-134. doi: 10.1037/a0021126

Mastandrea, S., and Maricchiolo, F. (2014). Implicit and explicit aesthetic evaluation of design objects. Art Percept. 2, 141-162. doi: 10.1163/2213491300002015

McManus, I. C., Jones, A. L., and Cottrell, J. (1981). The aesthetics of colour. Perception 10, 651-666. doi: 10.1068/p100651

McManus, I. C., Zhou, F. A., L'Anson, S., Waterfield, L., Stöver, K., and Cook, R. (2011). The psychometrics of photographic cropping: the influence of colour, meaning and expertise. Perception 40, 332-357. doi: 10.1068/ p6700

Meyers-Levy, J., and Zhu, R. (2007). The influence of ceiling height: the effect of priming on the type of processing that people use. J. Consum. Res. 34, 174-186. doi: $10.1086 / 519146$ 
Mühlenbeck, C., Liebal, K., Pritsch, C., and Jacobsen, T. (2016). Differences in the visual perception of symmetric patterns in orangutans (Pongo pygmaeus abelii) and two human cultural groups: a comparative eye-tracking study. Front. Psychol. 7:408. doi: 10.3389/fpsyg.2016.00408

Mühlenbeck, C. A., Liebal, K., Pritsch, C., and Jacobsen, T. (2015). Gaze duration biases for colours in combination with dissonant and consonant sounds: a comparative eye-tracking study with orangutans. PLoS ONE 10:e0139894. doi: 10.1371/journal.pone.0139894

Müller, M., Höfel, L., Brattico, E., and Jacobsen, T. (2010). Aesthetic judgments of music in experts and laypersons - An ERP study. Int. J. Psychophysiol. 76, 40-51. doi: 10.1016/j.ijpsycho.2010.02.002

Nasar. (1994). Urban design aesthetics: the evaluative qualities of building exteriors. Environ. Behav. 26, 377-401. doi: 10.1177/001391659402600305

Nieminen, S., Istók, E., Brattico, E., and Tervaniemi, M. (2012). The development of the aesthetic experience of music: preference, emotions, and beauty. Mus. Sci. 16, 372-391. doi: 10.1177/1029864912450454

Nieminen, S., Istok, E., Brattico, E., Tervaniemi, M., and Huotilainen, M. (2011). The development of aesthetic responses to music and their underlying neural and psychological mechanisms. Cortex 47, 1138-1146. doi: 10.1016/j.cortex. 2011.05.008

North, A. C. (2010). Individual differences in musical taste. Am. J. Psychol. 123, 199-208. doi: 10.5406/amerjpsyc.123.2.0199

Obermeier, C., Menninghaus, W., von Koppenfels, M., Raettig, T., SchmidtKassow, M., Otterbein, S., et al. (2013). Aesthetic and emotional effects of meter and rhyme in poetry. Front. Psychol. 4:10. doi: 10.3389/fpsyg.2013.00010

Orgs, G., Hagura, N., and Haggard, P. (2013). Learning to like it: aesthetic perception of bodies, movements and choreographic structure. Conscious. Cogn. 22, 603-612. doi: 10.1016/j.concog.2013.03.010

Penhune, V. B. (2011). Sensitive periods in human development: evidence from musical training. Cortex 47, 1126-1137. doi: 10.1016/j.cortex.2011.05.010

Perani, D., Saccuman, M. C., Scifo, P., Spada, D., Andreolli, G., Rovelli, R., et al. (2010). Functional specializations for music processing in the human newborn brain. Proc. Natl. Acad. Sci. U.S.A. 107, 4758-4763. doi: 10.1073/pnas. 0909074107

Petty, R. E., Priester, J. R., and Wegener, D. T. (1994). "Cognitive processes in attitude change," in Handbook of Social Cognition, 2nd Edn, eds R. S. Wyer and T. K. Srull (Hillsdale, NJ: Erlbaum), 69-142.

Petty, R. E., Wegener, D. T., and Fabrigar, L. R. (1997). Attitudes and attitude change. Annu. Rev. Psychol. 48, 609-647. doi: 10.1146/annurev.psych.48.1.609

Piaget, J. (1926). The Language and Thought of the Child. New York, NY: Harcourt, Brace.

Purcell, A. T., Peron, E., and Berto, R. (2001). Why do preferences differ between scene types? Environ. Behav. 33, 93-106. doi: 10.1177/00139160121972882

Rawlings, D. (2000). The interaction of openness to experience and schizotypy in predicting preference for abstract and violent paintings. Emp. Stud. Arts 18, 69-91. doi: 10.2190/71CT-AA49-XRMG-C842

Rawlings, D. (2003). Personality correlates of liking for 'unpleasant' paintings and photographs. Pers. Indiv. Differ. 34, 395-410. doi: 10.1016/S0191-8869(02) 00062-4

Rentfrow, P. J., and Gosling, S. D. (2003). The do re mi's of everyday life: the structure and personality correlates of music preferences. J. Pers. Soc. Psychol. 84, 1236-1256. doi: 10.1037/0022-3514.84.6.1236

Riemer, H., Shavitt, S., Koo, M., and Markus, H. R. (2014). Preferences don't have to be personal: expanding attitude theorizing with a cross-cultural perspective. Psychol. Rev. 121, 619-648. doi: 10.1037/a0037666

Ritterfeld, U. (2002). Social heuristics in interior design preferences. J. Environ. Psychol. 22, 369-386. doi: 10.1006/jevp.2002.0276

Röder, B., Ley, P., Shenoy, B. H., Kekunnaya, R., and Bottari, D. (2013). Sensitive periods for the functional specialization of the neural system for human face processing. Proc. Natl. Acad. Sci. U.S.A. 110, 16760-16765. doi: 10.1073/pnas. 1309963110

Roulston, K. (2006). Qualitative investigation of young children's music preferences. Int. J. Educ. Arts 7, 1-24.

Sachs, M. E., Damasio, A., and Habibi, A. (2015). The pleasures of sad music: a systematic review. Front. Hum. Neurosci. 9:404. doi: 10.3389/fnhum.2015.00404

Schank, R. C., and Abelson, R. P. (1977). Scripts, Plans, Goals and Understanding: An Inquiry into Human Knowledge Structures. Hillsdale, NJ: Erlbaum, 41.
Smith, J. D., and Melara, R. J. (1990). Aesthetic preference and syntactic prototypicality in music: 'Tis the gift to be simple. Cognition 34, 279-298. doi: 10.1016/0010-0277(90)90007-7

Stevens, C., Schubert, E., Haszard Morris, R., Frear, M., Chen, J., Healey, S., et al. (2009). Cognition and the temporal arts: Investigating audience response to dance using PDAs that record continuous data during live performance. Int. J. Hum. Comput. Stud. 67, 800-813. doi: 10.1016/j.ijhcs.2009. 06.001

Symons, D. (1995). "Beauty is in the adaptations of the beholder: the evolutionary psychology of human female sexual attractiveness," in Sexual Nature/Sexual Culture, eds P. R. Abramson and S. D. Pinkerton (Chicago, IL: University of Chicago Press), 80-118.

Tervaniemi, M., Ilvonen, T., Sinkkonen, J., Kujala, A., Alho, K., Huotilainen, M., et al. (2000). Harmonic partials facilitate pitch discrimination in humans: electrophysiological and behavioral evidence. Neurosci. Lett. 279, 29-32. doi: 10.1016/S0304-3940(99)00941-6

Tinio, P. P. L., and Leder, H. (2009). Natural scenes are indeed preferred, but image quality might have the last word. Psychol. Aesthet. Creat. Arts 3, 52-56. doi: $10.1037 / \mathrm{a} 0014835$

Tinio, P. P. L., Leder, H., and Strasser, M. (2011). Image quality and the aesthetic judgement of photographs: contrast, sharpness, and grain teased apart and put together. Psychol. Aesthet. Creat. Arts 5:165. doi: 10.1037/a0019542

Trainor, L. J. (2005). Are there critical periods for musical development? Dev. Psychobiol. 46, 262-278. doi: 10.1002/dev.20059

Trainor, L. J., Tsang, C. D., and Cheung, V. H. W. (2002). Preference for sensory consonance in 2- and 4-month-old infants. Music Percept. 20, 187-194. doi: $10.1525 / \mathrm{mp} .2002 .20 .2 .187$

Trehub, S. E. (2003). The developmental origins of musicality. Nat. Neurosci. 6, 669-673. doi: 10.1038/nn1084

Trehub, S. E. (2006). "Infants as musical connoisseurs," in The Child as Musician A Handbook of Musical Development, ed. G. E. McPherson (Oxford: Oxford University Press), 33-49.

Trehub, S. E., Schellenberg, G., and Hill, D. (1997). "The origins of music perception and cognition: a developmental perspective," in Perception and Cognition of Music, eds I. Deliege and J. A. Sloboda (Hove: Psychology Press), 103-128.

Van den Berg, A. E., Koole, S. L., and van der Wulp, N. Y. (2003). Environmental preference and restoration: (How) are they related? J. Environ. Psychol. 23, 135-146. doi: 10.1016/S0272-4944(02)00111-1

Vartanian, O., Navarrete, G., Chatterjee, A., Brorson Fich, L., Gonzalez-Mora, J. L., Leder, H., et al. (2015). Architectural design and the brain: effects of ceiling height and perceived enclosure on beauty judgments and approach-avoidance decisions. J. Environ. Psychol. 41, 10-18. doi: 10.1016/j.jenvp.2014.11.006

Vartanian, O., Navarrete, G., Chatterjee, A., Fich, L. B., Leder, H., Modroño, C., et al. (2013). Impact of contour on aesthetic judgments and approach-avoidance decisions in architecture. Proc. Natl. Acad. Sci. U.S.A. 110, 10446-10453. doi: 10.1073/pnas.1301227110

Wassiliwizky, E., Wagner, V., Jacobsen, T., and Menninghaus, W. (2015). Artelicited chills indicate states of being moved. Psychol. Aesthet. Creat. Arts 9, 405-416. doi: 10.1037/aca0000023

Weber, R. (1995). On the Aesthetic of Architecture: A Psychological Approach to the Structure and the Order of Perceived Architectural Space. Aldershot: Avebury.

Weber, R., Schnier, J., and Jacobsen, T. (2008). Aesthetics of streetscapes: influence of fundamental properties on aesthetic judgments of urban space. Percept. Mot. Skills 106, 128-146. doi: 10.2466/pms.106.1.128-146

White, E. J., Hutka, S. A., Williams, L. J., and Moreno, S. (2013). Learning, neural plasticity and sensitive periods: implications for language acquisition, music training and transfer across the lifespan. Front. Syst. Neurosci. 7:90. doi: 10. 3389/fnsys. 2013.00090

Whitfield, T. W. A. (1984). Individual differences in evaluation of architectural colour: categorization effects. Percept. Mot. Skills 59, 183-186. doi: 10.2466/ pms.1984.59.1.183

Whitfield, T. W. A., and Slatter, P. E. (1979). The effects of categorization and prototypicality on aesthetic choice in a furniture selection task. Br. J. Psychol. 70, 65-75. doi: 10.1111/j.2044-8295.1979.tb02144.x

Zajonc, R. B. (1968). Attitudinal effects of mere exposure. J. Personal. Soc. Psychol. Monogr. Suppl. 9, 1-27. doi: 10.1037/h0025848 
Zeki, S., Watson, J. D. G., Lueck, C. J., Friston, K. J., Kennard, C., and Frackowiak, R. S. J. (1991). A direct demonstration of functional specialization in human visual cortex. J. Neurosci. 11, 641-649.

Conflict of Interest Statement: The authors declare that the research was conducted in the absence of any commercial or financial relationships that could be construed as a potential conflict of interest.
Copyright (๑) 2017 Jacobsen and Beudt. This is an open-access article distributed under the terms of the Creative Commons Attribution License (CC BY). The use, distribution or reproduction in other forums is permitted, provided the original author(s) or licensor are credited and that the original publication in this journal is cited, in accordance with accepted academic practice. No use, distribution or reproduction is permitted which does not comply with these terms. 